\title{
Vickers micro-hardness variation during change in concentration of constituent elements in $\mathrm{Ni}_{50-x} \mathrm{Fe}_{x} \mathrm{Mn}_{30} \mathrm{Sn}_{20-y} I n_{y}$, Heusler alloys
}

\author{
Sandeep Nambiar S. ${ }^{1}$, Murthy B.R.N. ${ }^{1,}$, Sathyashankara S. ${ }^{1}$, Prasanna A.A. ${ }^{2}$, and Arout Chelvane J. \\ ${ }^{1}$ Department of Mechanical and Manufacturing Engineering, Manipal Institute of Technology, Manipal Academy of Higher \\ Education, Manipal, Karnataka 576104, India \\ 2 Department of Physics, Malnad College of Engineering, Hassan, Karnataka 573202, India \\ 3 Advanced Magnetics Laboratory, Defense Metallurgical Research Laboratory, Kanchanabagh, Hyderabad, Telangana 500066, \\ India
}

Received: 7 October 2021 / Accepted: 4 January 2022

\begin{abstract}
Present work is on Heusler alloys of the sequence $\mathrm{Ni}_{50-x} \mathrm{Fe}_{x} \mathrm{Mn}_{30} \mathrm{Sn}_{20-y} \mathrm{In}_{y}$, were prepared in order to investigate the relationship between microstructure and mechanical property. The work represents the variations in the hardness of the alloy when the component elements are changed. Alloys show Vickers hardness $\mathrm{HV}=3.5 \mathrm{GPa}$ at $x=2$ and $y=4$. At $x=4$ and $y=8$, alloy exhibits an $\mathrm{L} 1_{0}$ tetragonal structure, whereas at $x=3$ and $y=6 \mathrm{~L} 2{ }_{1}$ austenite phase structure is observed. Interface piling up occurs which greatly reduces fracture propagation and dislocation at neighboring interfaces. Large piled-up interfaces available in the martensite phase due to the sub-strips significantly contribute this process resulting in large hardness value. In spite of thicker laminates in the austenite phase, the alloy exhibits higher hardness than martensite phase or even the composite. Hardness is particularly low in the martensitic phase $(x=4, y=8)$, which is produced owing to interfacial motion. The hardness value falls as the Sn concentration increases due to weak pinning between the strips. A drastic increase in hardness of $3.5 \mathrm{GPa}$ has been observed when $x=2$ and $y=4$.
\end{abstract}

Keywords: Heusler alloy / vaccum arc melting / vickers hardness / martensitic transformation

\section{Introduction}

The current era's biggest environmental issues on a global scale is the decline of the ozone layer, which is caused by the overwhelming amount of humans reliance on vapour compression refrigeration and air conditioning systems [1]. Many refrigerators and air conditioners employ refrigerant gases known as chlorofluorocarbons (CFCs) [2]. The ozone layer has suffered greatly due to the many years exposure to these substances. Because of this, we have a great responsibility to create refrigeration systems that are free of chemicals and harmless to the environment [3]. One of the alternatives to existing refrigeration technology is magnetic refrigeration. Magnetic refrigeration systems are predicted to become more prevalent since they are a newer generation technology that releases no pollutants, unlike vapor-compression refrigeration systems [4-7]. More recently the usage of magnetic refrigerants has emerged in refrigeration procedures. Adiabatic demagnetization is used to magnetise

\footnotetext{
* e-mail: murthy.brn@manipal.edu
}

other materials. Magnetic refrigeration is performed by mixing a very hot material with a very powerful magnetic field source.

In diffusion-less process, the material transforms from its initial austenitic phase to a first-order martensitic transition. Because the material transitions between martensitic and austenitic states, it has a changeable ability to maintain certain qualities during deformation in its martensitic state and to transition back to an austenitic state after being heated.

Some types of Heusler alloys display features such as shape memory and magnetocaloric effects as the alloys undergo a martensitic transition. Alloy properties show good promise for industrial use. The following is about alloy $\mathrm{L} 1_{0}$ and $\mathrm{L} 2{ }_{1}$ structures in their martensitic and austenite phases [5]. The $\mathrm{Ni}_{50-x} \mathrm{Fe}_{x} \mathrm{Mn}_{30} \mathrm{Sn}_{20-y} \operatorname{In}_{y}(1 \leq x \leq 4 ; 2 \leq y \leq 8)$ alloy series is the subject of the current investigation. Applications look to get a boost since near-room-temperature martensitic transitions are of interest to alloys. The established alloy system features a room temperature martensitic transition and consists of a mix of nickel with anywhere from 30-40\% tin, 1-4\% manganese, and 1-8\% indium. Following a test for a martensitic transformation with the alloy's e/a ratio, the following alloys 
were generated [8-13]. Variation in e/a results in change of martensitic transformation and also phase change associated with it [14].

The valence electrons per atom for $\mathrm{Ni}, \mathrm{Mn}, \mathrm{Sn}, \mathrm{Fe}$, and In are, respectively, three, four, five, eight, and three. Electron concentrations in the outer shells can be calculated according to the equation shown here [14].

\section{See equation (1) below.}

Equation (1) is used to tune the composition of the alloys systems to near room temperature and to keep the $\mathrm{e} / \mathrm{a}$ of the alloy between 7.80 and 7.70. A temperature range of 800 to $900^{\circ} \mathrm{C}$ must be maintained during annealing in order for the martensitic transformation to take place in the material and for the material to show signs of phase transition [15]. When it comes to preventing oxidation on the specimen's surface, the vast majority of studies recommend that the specimen be annealed in a vacuum atmosphere. Grain boundaries for the sample while working on a $\mathrm{Mn}_{2} \mathrm{FeGe}$ Heusler alloy for $30 \mathrm{~s}$ with Marble's reagent was seen. It was discovered via research on NiMnGa-Co-based Heusler alloys that the microstructure of the alloys may be determined by employing Marble's reagent as an etchant. Additionally, the Marble's reagent was used to analyse the principal makeup of numerous additional works including one in which the major composition of Heusler alloys was Ni-Mn-Sn [16-23].

\section{Experimental section}

\subsection{Material preparation}

$5 \mathrm{~g}$ weight ingots were produced by vacuum arc melting furnace in an argon environment, alloying elements of $\mathrm{Ni}, \mathrm{Sn}$, $\mathrm{Mn}, \mathrm{In}$ and $\mathrm{Fe}$ of $99.99 \%$ were taken to produce the master alloy. The temperature of the copper hearth and electrodes is lowered by utilizing chiller water. After the master alloys have been completely melted, they are hardened. These are then re-melted by rotating them using the tweeze mechanism. A homogenous alloy is formed by the continuous melting, re-melting and solidification process that are repeated and thus the alloys form a homogeneity.

The formed alloys were then annealed at $750{ }^{\circ} \mathrm{C}$ in a vacuum setup to prevent surface oxidation for $48 \mathrm{~h}$. A small section from the master alloy was cut by electrical discharge machining, was utilized to analyses the X-ray Diffraction (XRD) pattern of samples. The XRD patterns from the alloys were recorded using a high-resolution X-ray diffractometer (X'Pert PRO PANalytical) with filtered $\mathrm{CuK}$ radiation of $0.15410 \mathrm{~nm}$. A curved graphite monochrometer was used to run the diffractometer at $40 \mathrm{kV}$ and $30 \mathrm{~mA}$. A continuous $\theta-2 \theta$ scanning was performed across the diffraction angle $2 \theta$ in the $10^{\circ}<2 \theta<90^{\circ}$ range, which

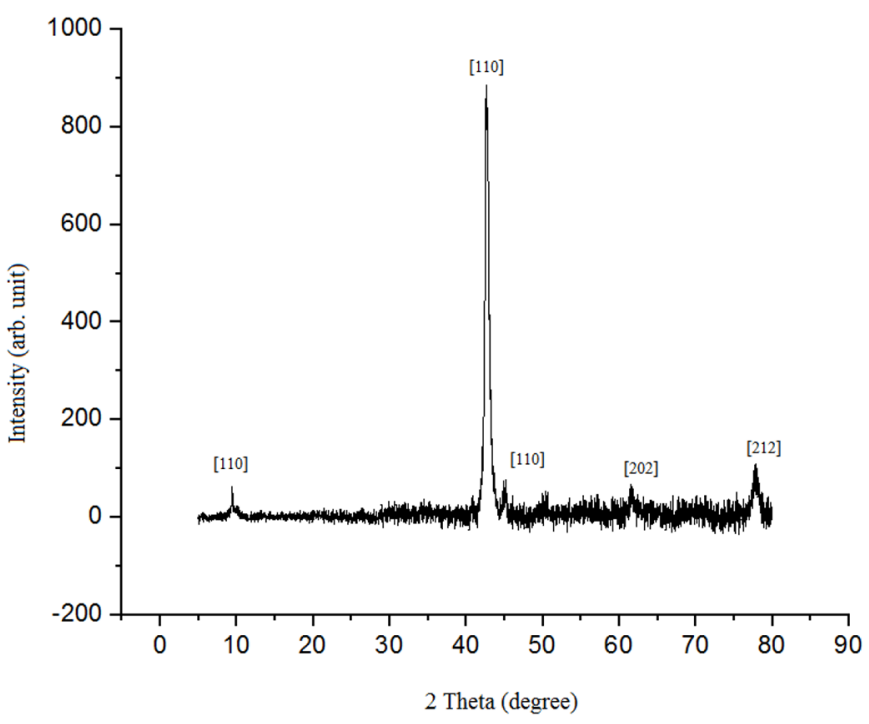

Fig. 1. XRD pattern in $\mathrm{Ni}_{49} \mathrm{Fe}_{1} \mathrm{Mn}_{30} \mathrm{Sn}_{18} \mathrm{In}_{2}$ alloy.

covers the range of the typical diffraction peaks. The data were collected at room temperature and at varying temperatures. The results were collected at ambient temperature as well as at selected temperatures ranging from 24.85 to $126^{\circ}$. High temperature investigations at various temperatures enabled the determination of an alloys lattice expansion and/or phase change from a martensite to an austenite structure. For the various alloy samples, step scans was completed with a range of $35^{\circ}<2 \theta$ $<70^{\circ}$ at a scan rate of $0.02^{\circ} / \mathrm{min}$ and $2-4 \mathrm{~s}$. The microstructure imaging was done using Field Emission Scanning Electron Microscope (FESEM) Carl ZEISS make GeminiSEM 300 with probe current of $3 \mathrm{pA}$ to $20 \mathrm{nA}$.

At the upper (U), lower (L) and central (C) positions of the alloy Vicker's hardness (HV) was measured to see whether there is a relationship between compositional dependency and mechanical hardness of the alloy. The HV was measured with a Vickers hardness tester under a force of $200 \mathrm{kgf}$. A force of 1 to $200 \mathrm{kgf}$ is applied to a diamond depression at an angle of $136^{\circ}$ between opposing sides. This is done between 10 and $15 \mathrm{~s}$ of dwell time.

\section{Results and discussion}

$\mathrm{Ni}_{49} \mathrm{Fe}_{1} \mathrm{Mn}_{30} \mathrm{Sn}_{18} \mathrm{In}_{2}$ alloy was split in half and annealed at $750^{\circ} \mathrm{C}$, while the other was as-cast Heusler alloy and further subjected to XRD analysis. This was done in order to estimate the influence of annealing process on the properties of the Heusler alloy. The XRD patterns for the alloys were recorded in the range and the diffraction angle $2 \theta$ is shown in Figure 1 which shows that as the alloying elements composition is changed there tends to be a change in the peaks as well.

$$
e / a=\frac{10 x(\text { Niat. } \%)+7 x(\text { Mnat. } \%)+4 x(\text { Snat. } \%)+8 x(\text { Feat. } \%)+3 x(\text { Inat. } \%)}{100}
$$


Table 1. Vickers micro hardness measured for the alloy at Upper (U), central (C) and lower (L) cross section of $\mathrm{Ni}_{50-x} \mathrm{Fe}_{\mathrm{x}} \mathrm{Mn}_{30} \mathrm{Sn}_{20-\mathrm{y}} \mathrm{In}_{\mathrm{y}}(1 \leq \mathrm{x} \leq$ alloy $4 ; 2 \leq \mathrm{y} \leq 8)$.

\begin{tabular}{|c|c|c|c|c|}
\hline \multicolumn{5}{|c|}{$H V(G P a)$} \\
\hline $\bar{X}$ & $Y$ & $U$ & C & $L$ \\
\hline 1 & 2 & 2.503 & 3.048 & 2.617 \\
\hline 2 & 4 & 3.112 & 3.520 & 3.227 \\
\hline 3 & 6 & 2.105 & 2.701 & 2.254 \\
\hline 4 & 8 & 2.617 & 2.704 & 2.716 \\
\hline
\end{tabular}

The X-ray diffraction pattern (illustrated in Fig. 1) obtained at room temperature reveals that the sample exhibits the Heusler crystal structure in as-cast alloy. There is also evidence of peak splitting which occurred at the most extreme peak. This also indicates that the specimen has undergone martensitic transformation. Although, when the peak is enlarged, it is evident that there is solid indication of peak splitting caused by the martensitic transformation. The numerous splits-like pattern displayed may have evolved as a result of the main split visible. While there is evidence of peak splitting in the as cast specimen, it cannot be considered convincing proof. The combined XRD results of alloys represented as A, B, C and D respectively as seen in Figure 2 also indicate the same.

Figure 3a depicts the microstructure of an annealed specimen. To break down the dendritic structure, the specimens are annealed in a furnace for $48 \mathrm{~h}$. During annealing, which involves heating to a high temperature for an extended period of time, the diffusion kinematics is triggered at a higher pace, annihilating the dendritic structure and resulting in a well-distributed grain structure.

The coarser dendrites appear to be fractured, revealing fine grain, well distributed phases. Figure $3 \mathrm{~b}$ depicts the microstructure of the as cast Heusler alloy. It depicts the dendritic structure of both phases, with coarser dendrites. The two stages appear to be fairly evenly distributed. The quantity of the second phase (phase 2) appears to be less than that of the first. Dendrites have inhomogeneous chemical compositions at the microscopic level from place to location or dendrite to dendrite. The characteristics of the dendritic structure are usually inhomogeneous throughout.

According to the Vickers hardness test findings, the bulk structure is a primary austenite phase that is softer in nature and has been cooled at a quicker pace as in Figure 4 .

The hardness that propagates with the local-strain acts as a carrier in the microscopic martensite strips, which break apart and self-adjust in a changed pattern, as depicted. Free energy at martensite strip interfaces is larger than the free energy in the interior of martensite strips. In the strengthening of small crystallite alloys, interfaces prevent dislocation motion. Large piled-up interfaces accessible in the martensite phase as a result of nano crystallite sub strips play a significant role in this process, resulting in a high average $\mathrm{HV}$ value at the upper cross section. For $x$ and $y$ are reduced, the hardness falls to 2.264 from $2.736 \mathrm{GPa}$ when $x=4$ and $y=8$. When $x$ and $y$ are decreased to 2 and 4, respectively, the greatest hardness value of $3.53 \mathrm{GPa}$ is attained from $3.058 \mathrm{GPa}$. At $x=4$ and $y=8$, we find a martensitic $\mathrm{L} 1_{0}$ tetragonal structure, while at $x=3$ and $y=6$, we see an

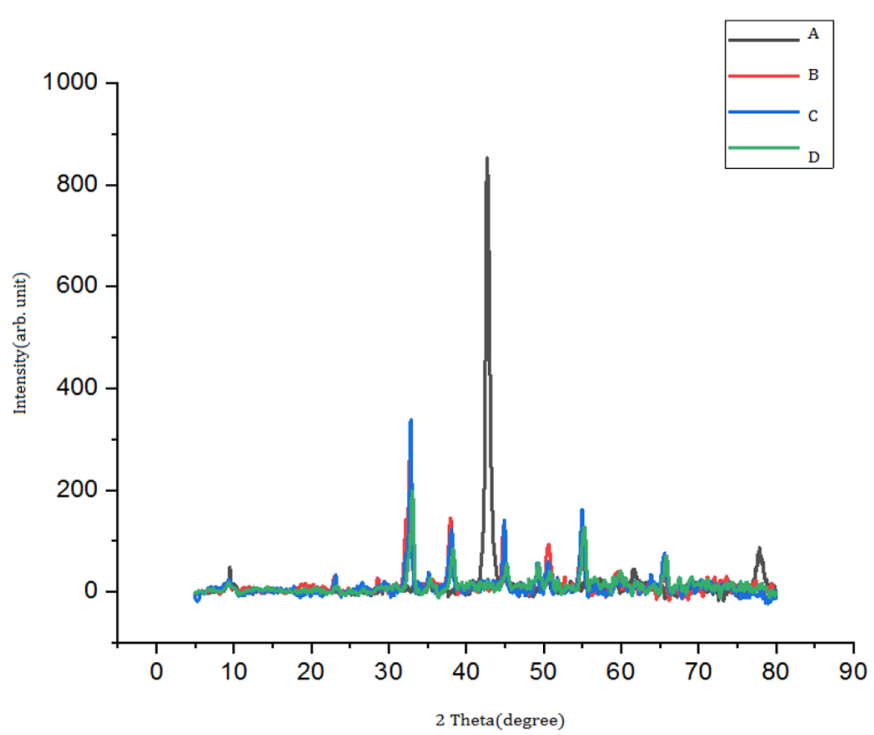

Fig. 2. XRD patterns of alloys $\mathrm{Ni}_{49} \mathrm{Fe}_{1} \mathrm{Mn}_{30} \mathrm{Sn}_{18} \mathrm{In}_{2}$, $\mathrm{Ni}_{48} \mathrm{Fe}_{2} \mathrm{Mn}_{30} \mathrm{Sn}_{16} \mathrm{In}_{4}, \quad \mathrm{Ni}_{47} \mathrm{Fe}_{3} \mathrm{Mn}_{30} \mathrm{Sn}_{14} \mathrm{In}_{6}$ and $\mathrm{Ni}_{46} \mathrm{Fe}_{4} \mathrm{Mn}_{30}$ $\mathrm{Sn}_{12} \mathrm{In}_{8}$ represented as A, B, C and D respectively.

austenite $\mathrm{L} 2{ }_{1}$ phase. The alloy is strengthened, particularly during the martensite phase, since the free energy at the interface between the strips is greater than in the inner area of the strips. This happens in martensite which separates into thinner laminates and so piles up, and it significantly reduces fracture propagation and dislocation at neighboring surfaces. Despite the thicker laminates in the austenite phase, the hardness is greater than in the martensite phase or even the composite. Hardness is particularly low in the martensite phase $(x=4, y=8)$, which is produced via interfacial motion. The hardness value decreases with increasing Sn content due to the process of weak pinning creation between the strips, indicating better interfacial motion with additional weaker pinning due to higher shear stress between the strips. Because there are no M-M or M-A contacts in the austenite alloy $(x=2, y=4)$, the hardness rises dramatically to $3.5 \mathrm{GPa}$. The super-lattice structure is thought to be the cause of the significant drop in hardness in the other austenite alloy $(x=1$, $y=2$ ). Energy Dispersive X-Ray Analysis (EDX) analysis technique was used to determine the composition of the alloy and Table 2 depicts the composition of the alloy at varying $\mathrm{x}$ and $\mathrm{y}$ values of $\mathrm{Ni}_{50-x} \mathrm{Fe}_{x} \mathrm{Mn}_{30} \mathrm{Sn}_{20-y} \mathrm{In}_{y}(1 \leq \mathrm{x} \leq 4 ; 2 \leq \mathrm{y} \leq 8)$ alloy. The results envisage the homogenous alloying all the four composition. 

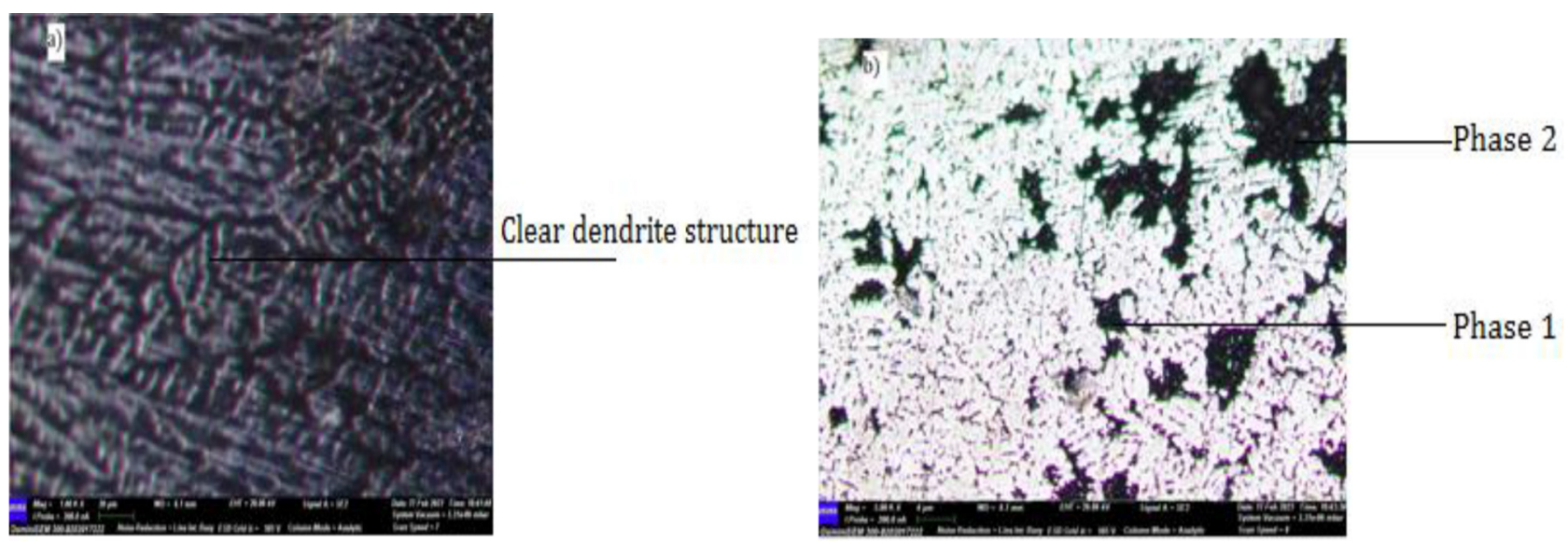

Fig. 3. (a) FESEM image of annealed $\mathrm{Ni}_{49} \mathrm{Fe}_{1} \mathrm{Mn}_{30} \mathrm{Sn}_{18} \mathrm{In}_{2}$ alloy; (b) FESEM image of as-cast $\mathrm{Ni}_{49} \mathrm{Fe}_{1} \mathrm{Mn}_{30} \mathrm{Sn}_{18} \mathrm{In}_{2}$ alloy.

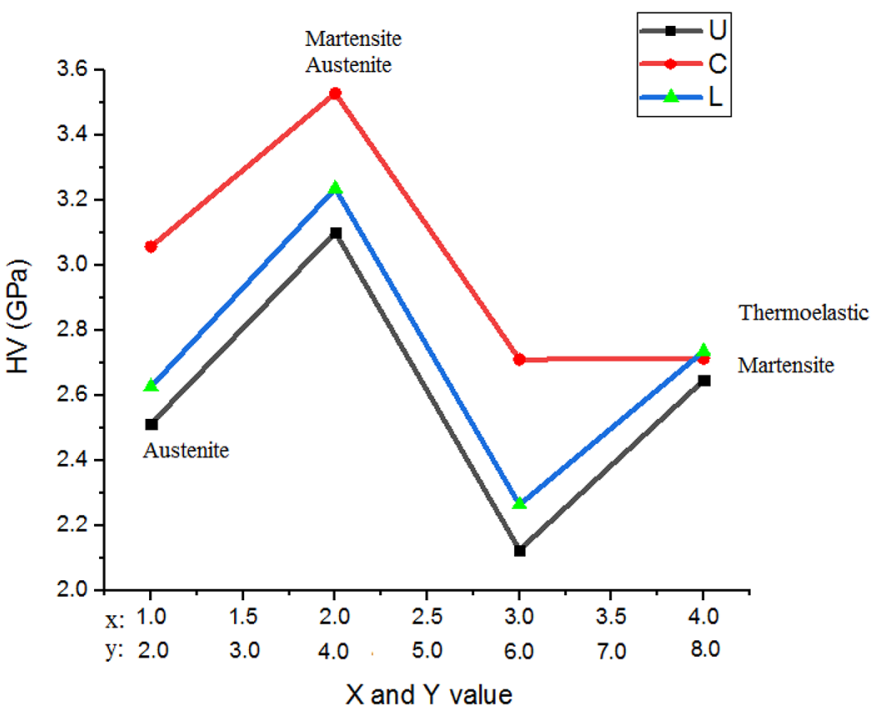

Fig. 4. Vickers micro hardness measured on upper (U), central (C) and lower (L) cross-sections from an alloy $\mathrm{Ni}_{50-x} \mathrm{Fe}_{x} \mathrm{Mn}_{30} \mathrm{Sn}_{20-y} \mathrm{In}_{y}$ $(1 \leq x \leq 4 ; 2 \leq y \leq 8)$.

Table 2. Compositional analysis of arc melted $N i_{50-x} F e_{x} M n_{30} \operatorname{Sn}_{20-y} \operatorname{In}_{y}(1 \leq \mathrm{x} \leq 4 ; 2 \leq \mathrm{y} \leq 8)$ alloy.

\begin{tabular}{lllllll}
\hline & \multicolumn{5}{c}{ Composition (wt \%) } \\
\hline$x$ & $y$ & $\mathrm{Ni}$ & $\mathrm{Mn}$ & $\mathrm{Sn}$ & $\mathrm{In}$ & $\mathrm{Fe}$ \\
\hline 1 & 2 & 37 & 26 & 31 & 3 & 0.7 \\
2 & 4 & 37 & 26 & 27 & 7 & 1.1 \\
3 & 6 & 37 & 26 & 23 & 10 & 1.3 \\
4 & 8 & 36 & 26 & 13 & 1.7 \\
\hline
\end{tabular}

The values are accurate within $\pm 0.1 \%$ error. 


\section{Conclusions}

A Ni-Mn based Heusler alloys have been synthesised and the desired results have been obtained. The bulk structure is a main austenite phase that is softer in nature and has been cooled faster, local-strain hardness acts as a carrier in the small martensite strips that break apart and selfaccommodate in a changed pattern. An increasing temperature gradient governs the evolution of the microstructure in martensite strips of small crystallites in response to atom dynamics at bulk alloy surfaces. Modifying the microstructure prevents crack formation and propagation. The free energy at martensite interfaces is higher than the free energy within martensite strips. Interfaces slow dislocation motion in small crystallite alloys. High average $\mathrm{HV}$ value at higher cross section is due to large piled-up interfaces accessible in martensite phase due to nano crystallite sub strips. Since free energy is higher at the interface between the strips than the interior area of the strips, the alloy is reinforced. Despite the thicker laminates, austenite is harder than martensite or even composite. The martensite phase $(x=4, y=8)$ has the lowest hardness. Due to less pinning between the strips, the hardness value falls with increasing Sn concentration, suggesting improved interfacial mobility with increased shear stress between the strips. The absence of M-M or M-A contacts in the austenite alloy $(x=2, y=4)$ raises the hardness to $3.5 \mathrm{GPa}$. The second austenite alloy $(x=1, y=2)$ has a substantial decrease in hardness due to the super-lattice structure.

\section{References}

1. O. Secretariat, Montreal Protocol on Substances That Deplete The Ozone Layer Recognition of Some of the Exemplary Projects That Have Been Undertaken Pursuant to Article 10 of the Montreal Protocol Ozone Secretariat (2007)
2. S.A. Montzka, G.S. Dutton, P. Yu, CFC-11 55 (2018) 413-417

3. N.A. Mezaal, K.V. Osintsev, T.B. Zhirgalova, IOP Conf. Ser. Earth Environ. Sci. 87 (2017) 032024

4. V.K. Pecharsky, Magnetocaloric and magnetic refrigeration, J. Magn. Magn. Mater. J. 200 (1999) 44-56

5. T. Krenke, M. Acet, E.F. Wassermann, X. Moya, L. Mañosa, A. Planes, Physical Rev. B 72 (2005) 014412

6. A. Nespoli, C.A. Biffi, E. Villa, A. Tuissi, J. Alloys Compd. 690 (2017) 478-484

7. G. Pirge, C.V. Hyatt, S. Altıntas, J. Mater. Process. Technol. 155 (2004) 1266-1272

8. C.O. Aguilar-Ortiz, D. Soto-Parra, Acta Mater. 107 (2016) $9-16$

9. H. Zhang, M. Qian, X. Zhang, J. Alloys Compd. 689 (2016) 481-488

10. X. Tian, Z. Wang, J. Zhu, C. Tan, K. Zhang, Z. Yu, W. Cai, J. Non Cryst. Solids. 495(2018) 19-26

11. H. Zhang, X. Zhang, M. Qian, L. Wei, D. Xing, J. Sun, L. Geng. J. Alloys Compd. 715 (2017) 206-213

12. X. Chen, V.B. Naik, R. Mahendiran, R.V. Ramanujan, J. Alloys Compd. 5 (2015) 097116

13. P. Bag, R. Nath, Solid State Commun. 270 (2018) 54-57

14. T. Krenke, E.F. Wassermann, X. Moya, L. Mañosa, A. Planes, Phys. Rev. B 73 (2006) 174413

15. G. Niu, C. Si, J. Jiao, Q. Han, M. Guo, M. Li, J. Alloys Compd. 830 (2020) 154693

16. G.A. Malygin, Solid State Phys. 61 (2019) 1251-1258

17. L. Huang, D.Y. Cong, Y. Ren, K.X. Wei, Y.D. Wang, Intermetallics 119 (2020) 1067036

18. S. Aksoy, M. Acet, P.P. Deen, L. Mañosa, A. Planes, Phys. Rev. B 79 (2009) 212401

19. S.V. Pedersen, J.R. Croteau, N. Kempf, Y. Zhang, D.P. Butt, B.J. Jaque, J. Solid State Chem. 285 (2020) 121203

20. Y. Zhang, J. Guo, M. Zhong, W. Xiang, Z. Wu, J. Magn. Magn. Mater. 521 (2021) 167540

21. F.X. Hu, B.G. Shen, J.R. Sun, Appl. Phys. Lett. 76 (2000) 14191

22. T. Chabri, A. Ghosh, S. Nair, A.M. Awasthi, A. Venimadhav, T.K. Nath, J. Phys. D: Appl. Phys. 51 (2018) 195001

23. K. Zhang, C. Tan, W. Zhao, E. Guo, X. Tian, ACS Appl. Mater. Interfaces 11 (2019) 34827-34840

Cite this article as: Sandeep Nambiar S., Murthy B.R.N., Sathyashankara S., Prasanna A.A., Arout Chelvane J. Vickers microhardness variation during change in concentration of constituent elements in $\mathrm{Ni}_{50-x} \mathrm{Fe}_{x} \mathrm{Mn}_{30} \mathrm{Sn}_{20-y} \mathrm{In}_{y}, \mathrm{Heusler}$ alloys, Manufacturing Rev. 9, 4 (2022) 